\title{
Quantifying the Effectiveness of Patterning, Test Conditions, and DIC Parameters for Characterization of Plastic Strain Localization
}

\author{
A. Forsström ${ }^{1} \cdot$ S. Bossuyt ${ }^{1} \cdot$ G. Scottii ${ }^{2} \cdot$ H. Hänninen ${ }^{1}$
}

Received: 1 June 2018 / Accepted: 5 April 2019 /Published online: 30 April 2019

(C) The Author(s) 2019

\begin{abstract}
Digital image correlation, commonly referred to as DIC, enables full-field measurements of displacements and strains from a surface of interest. While DIC offers major advantages over commonly established methods, such as strain gauges or extensometers, some questions remain in guaranteeing data precision of the DIC measurements. This paper reports on improvements in the precision of DIC measurements of large deformations in copper tensile specimens by optimized patterning and lighting conditions, as well as quantifying the improvements in the data. In the context of detection of strain localization at welds in copper canisters for spent nuclear fuel disposal, the trade-off between spatial resolution and strain resolution is critical. To quantify the improvements in measurement conditions, we propose a method for estimation of spatial resolution and noise level of a full-field strain measurement, which does not require imposing a displacement field with variations in strain or displacement of well-defined length scale.
\end{abstract}

Keywords Digital image correlation $\cdot$ Patterning $\cdot$ Spatial resolution $\cdot$ Noise $\cdot$ Strain localization

\section{Introduction}

Techniques using pattern matching in digital images, commonly referred to as Digital Image Correlation (DIC), are widely used for measuring shape, motion, and deformation of objects [1]. The major benefit of DIC is that it is not limited to point measurements, like strain gauges, or average measurements like extensometers are. Quantitative error analysis [2-6] of DIC technique shows that the measurement error depends critically on the presence of intensity gradients in the image. Therefore, obtaining satisfactory results with the technique often requires a sample preparation procedure to enhance image texture. Various methods for generating suitable textures at microscopic length scales have been reported in detail [7-9].

At macroscopic length scales, a suitable texture can often be achieved by spraying paint speckles onto the

A. Forsström

antti.forsstrom@aalto.fi

1 School of Engineering, Department of Mechanical Engineering, Engineering Materials, Aalto University, P.O. Box 14200, FI-00076 Aalto, Finland

2 School of Electrical Engineering, Nanofabrication Centre, Aalto University, Espoo, Finland object or by applying a given reproducible pattern with a suitable method, such as adhesive foils. When deformation is measured, the pattern has to deform with the surface of the object in order to give reliable information from the real strains. Therefore, the pattern has to be well attached to the surface of the specimen. With large strains paint or adhesive foil may detach from the surface or deform differently from the base material resulting in unreliable DIC data.

The material studied herein is phosphorous-alloyed oxygen-free copper (Cu-OFP) used for spent nuclear fuel disposal canisters [10]. Copper is a ductile material, which leads to large strains in tensile testing. Therefore, using paint or adhesive foils for patterning is not optimal, because the foil might detach or deform differently from the underlying material. To overcome this problem, the copper surface was oxidized with black copper oxide and the surface was patterned by etching an optimized DIC pattern [11] with the aid of a photolithographic method. The simplest solution, on the other hand, is to use the electro-discharge machined (EDM) surface directly as a natural pattern. Reflections from the roughness of the surface were found consistent enough for the sum-of-differential correlation to work, but the noise level of the data becomes rather high. In comparison to that, an optimized pattern improves the precision of the measurements considerably by providing easily trackable consistent features on the surface. 
In addition to methods for patterning objects for the purpose of DIC measurements, methods for characterizing the resulting patterns [12-14], and for choosing the analysis parameters in function of the characteristics of the pattern [15, 16] or in function of the strain field [17] have been proposed. However, some questions remain about guaranteeing the reliability of DIC measurements in terms of spatial resolution, accuracy, precision, sensitivity, and robustness of the measurement. It is well established, that there is a trade-off between spatial resolution and precision, which governs the choice of subset size when analyzing DIC measurements and which is affected by the quality of the pattern $[12,13]$. However, determination of spatial resolution of a measurement is not that straight-forward and often requires à priori tests with the same test conditions as the actual tests or imposing a synthetic displacement field with known variations in displacement [3-5, 18]. We propose, that in the absence of images with known imposed displacements, the autocorrelation length of the noise in the measurement may serve as a metric of the spatial resolution, since the wave length of the noise correlates with the spatial resolution of the measurement. The obtained value includes the combined effects of the test conditions and calculation methods, without any prior knowledge about those conditions or methods in advance. The autocorrelation length was extracted for a set of experimental images, in the context of detection of strain localization at copper welds, and for Sample 14 of the DIC Challenge [18], which contains known sinusoidally varying displacements.

\section{Experimental}

\section{Tensile Specimen Preparation and Naming}

In total, three $\mathrm{Cu}-\mathrm{OFP}$ tensile specimens were cut with electro-discharge machining (EDM) across an electron beam weld (EBW) and across a friction stir weld (FSW), see Fig. 1. Two of the specimens were cut next to each other from an electron beam welded copper plate. One of them was used as a reference specimen with the EDM-cut surface used as a natural DIC pattern (Fig. 1a) and the other one was patterned with the copper oxide pattern (Fig. 1b). From hereafter, the specimens are named EBW-EDM-S and EBW-P-S, respectively. $\mathrm{P}$ stands for patterned and $\mathrm{S}$ for spot lighting. A third specimen was cut across an FSW copper canister weld and patterned similarly to EBW-P-S, but in this instance indirect diffuse lighting was used instead of spot lighting (Fig. 1c). The specimen is designated as FSW-P-D, where D stands for diffuse lighting. The dimensions of the gauge length of the EBW specimens were $3.5 \times 25 \times 40 \mathrm{~mm}$, and the dimensions of the FSW specimen were $3.0 \times 30 \times 53.6 \mathrm{~mm}$.

\section{Pattern Generation}

The pattern bitmap was generated by specifying the spatial frequency content, with randomized phase, and calculating the inverse Fourier transformation as described in ref. [11]. The specified frequency content is directly related to the desired size of the pattern features, which was optimized by taking into consideration the imaging area of the specimen throughout the deformation. In other words, the camera had to be at a right distance from the specimen in order to see the whole specimen after deformation with the lens that was used. The resolution of the camera and lens system was evaluated at this set distance and taken into consideration during pattern generation so that the physical feature size on the specimen surface was close to 3 pixels in the image.

According to iDICs's Good Practice guide for DIC, a feature of a traditional speckle pattern should appear as 3-5 pixels on the CCD of the camera [19]. This is to minimize aliasing from smaller features ( $<3$ pixels), adding up as noise, and loss of spatial resolution from larger features ( $>5$ pixels). The pattern used herein solves the problem associated with speckle patterns by introducing evenly spaced features with varying shape, that are all within the desired size range, enabling consistent information from the whole field-of-view.

\section{Copper Oxide Pattern Preparation}

Before patterning, the EDM cut surface of the tensile specimens was polished with wet grinding and diamond paste polishing up to $1 \mu \mathrm{m}$ to allow an evenly thick copper oxide layer to be formed. An opaque layer of black copper(II)oxide $(\mathrm{CuO})$ was formed by treatment in $100 \mathrm{~g} / \mathrm{l}$ of 50/50 mixture of sodium hydroxide $(\mathrm{NaOH})$ and sodium chlorite $\left(\mathrm{NaClO}_{2}\right)$ at $85^{\circ} \mathrm{C}$ for about $10 \mathrm{~min}$. AZ5214E photoresist was then spincoated on top of the copper oxide layer. The spinning parameters were carefully adjusted for slow acceleration and deceleration, because the specimen was heavy and had an elongated shape. Even so it had to be well attached to the spinner. Maximum spin rate was about 800 RPM resulting in resist thickness of about $4 \mu \mathrm{m}$.

The photomask for pattern exposure was created with a Microtech LW405 laser writer using bitmap-patterning mode. The pattern was inscribed with the laser writer on a soda-lime glass plate which had a thin chromium layer. The pattern was transferred to the photoresist by placing the photomask directly on top of the copper specimen, onto which the photoresist was spin-coated, and the pattern was exposed with UV light from a mercury lamp. This method introduces a slight loss in optical resolution, but given the size of the features $(>150 \mu \mathrm{m})$, the loss is irrelevant. After exposure, the photoresist was developed in AZ351B solution; a NaOH-based developer, and finally the cupric oxide was patterned by etching 
Fig. 1 a) EBW-EDM-S; electron beam welded, EDM-cut surface used as a natural pattern, and direct spot lighting b) EBW-P-S; electron beam welded, patterned, and direct spot lighting c) FSW-P$\mathrm{D}$; friction stir welded, patterned, and indirect diffuse lighting. An approximate location of the welds is marked with orange lines

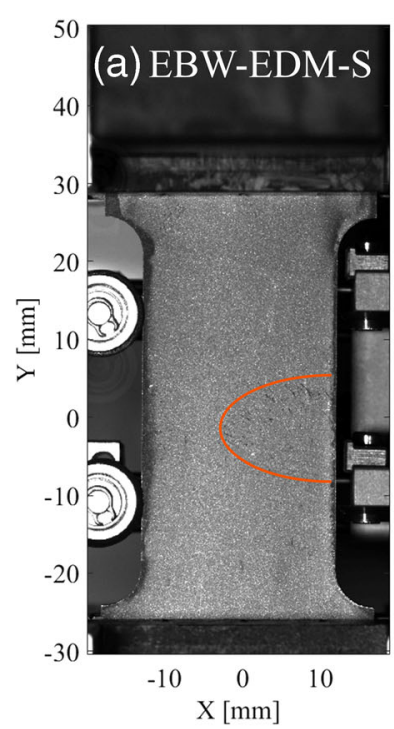

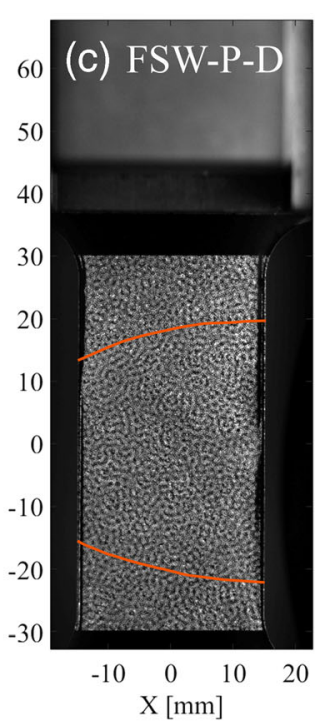

in $2 \% \mathrm{HCl}$ solution for a short time. The remaining photoresist was rinsed away with acetone.

Figure 2 shows optical micrographs of the EDM cut surface and the copper oxide patterned surface with the same magnification. The feature size of the pattern is relatively large when compared to the roughness of the EDM cut surface, but the EDM cut surface still works as a simple DIC pattern in 2D measurement, even if it is not optimal. The grains of copper are visible in the etched regions of the patterned surface.

\section{Tensile Testing with DIC}

Tensile testing with 2D optical displacement measurement through digital image correlation (DIC) was performed with a Zwick/Roell Z020 tensile testing machine and a StrainMaster DIC system by LaVision. The camera was a LaVision Imager pro $\mathrm{X}$ equipped with a Nikon MicroNikkor $105 \mathrm{~mm}$ lens. Dynamic range of the camera is 14 bits. The size of the camera sensor is $1600 \times 1200$ pixels, but the test images were taken with a narrowed field of view of roughly $600 \times 1600$ pixels. Physical size of the pixels was about $50 \mu \mathrm{m} /$ pixel for the EBW tests and $60 \mu \mathrm{m} /$ pixel for the FSW test due to slightly different camera distance from the specimen. Images were taken every $250 \mathrm{~s}$, but only every 20th image was used for the digital image correlation. This is equal to frame rate of one image per $1.4 \mathrm{~h}$ and cross-head displacement of $0.08 \mathrm{~mm}$ of the tensile testing machine. The tests were performed with constant extension rate of $0.001 \mathrm{~mm} /$ min, which corresponds to a strain rate of $4.17 \times 10^{-7} 1 / \mathrm{s}$ for gauge length of $40 \mathrm{~mm}$ and $3.1 \times 10^{-7} 1 / \mathrm{s}$ for gauge length of $53.6 \mathrm{~mm}$. The very low extension rate led to test duration of about two to three weeks depending on the specimen.

Two different lighting configurations were used in different experiments; 1) a direct spotlight from in front of the specimen (EBW-EDM-S, EBW-P-S) and 2) indirect diffused light (FSWP-D), where the spotlight was placed behind the specimen and the light was reflected from two curved curtains, made of projector screen canvas, located in front of the specimen (details can be found in ref. [20]). The monitoring of the specimen was performed from an opening between the curtains.

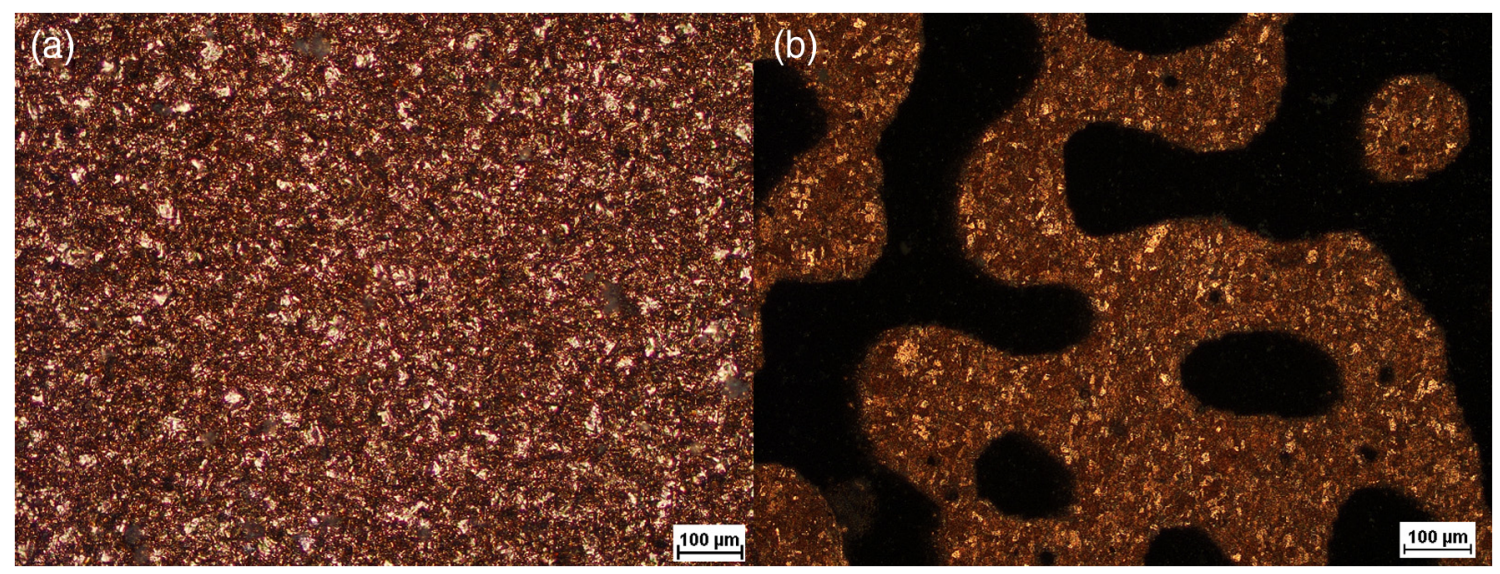

Fig. 2 a) EDM cut surface and b) copper oxide patterned surface 
Digital image correlation was performed repeatedly with LaVision DaVis software version 8.4.0 with varying calculation settings (Table 1). Since the trade-off between displacement precision and spatial resolution in DIC measurements is especially relevant, the subset size was systematically varied from 13 to 37 in a logarithmic series, roughly doubling the number of pixels per subset at each step in the series. Gaussian weighted round subsets were used, which affects the characteristics of the spatial resolution of the measurement.

\section{Data Analysis and Visualization}

\section{Strain Noise and Autocorrelation Length}

Displacement data obtained with DaVis software was transferred to Matlab for further analysis and visualization. The vertical engineering strain components were calculated by central finite differences on raw displacement field data. The edges of the displacement data were omitted to avoid erroneous strains from edge effects. No out-of-plane compensation was performed, which results in small errors in strain when necking of the specimen occurs. Since the plastic strains in these experiments are on the order of $50 \%$ or larger, this error is acceptable ( $0.05 \%$ estimated maximum apparent strain from out of plane component of the displacement field at final fracture zone).

Data accuracy and precision were assessed by calculating statistics for a section of the displacement field data that could be reasonably approximated as having uniform strain. Since the strain field was not perfectly uniform, the underlying trend was approximated by local regression smoothing (Lowess smoothing) over an area much larger than the subset size, and subtracted from the calculated strain field. A typical result of this procedure is shown in Fig. 3a). The remaining strain field thus can be considered as the noise in the DIC strain measurement. Its standard deviation quantifies the strain noise level, or the strain detection limit for the given experiment conditions and DIC calculation settings.
Quantifying the spatial resolution of the measurement is not as straightforward. There is clearly an intrinsic length scale to the noise in the DIC strain measurement, obtained by the above procedure. How well that length scale corresponds to the concept of spatial resolution, with which the displacement field or strain field is measured, is still somewhat of an open question, but in the absence of an imposed displacement field with variations in strain or displacement of well-defined length scale, the autocorrelation length of the noise in the measurement may serve as an upper bound on the spatial resolution of the measurement. There may be extraneous noise that is not subject to the smoothing effect inherent to DIC calculations with subsets, but if so the measurement can trivially be improved by smoothing the displacement field. The intrinsic length scale of the noise includes the combined effects of the subset size and strain calculation method, as well as those of the feature size distribution of the pattern. If the point spread function for the displacement field, calculated using Gaussian weights to emulate "round" subsets on a vertically stretched grid, is a 2-dimensional Gaussian function, with characteristic width $\boldsymbol{a}$ horizontally and $\boldsymbol{b}$ vertically, the expected point spread function for the vertical component of the strain field calculated from this displacement field is:

$z=\left(1-\frac{2 y^{2}}{b^{2}}\right) e^{-\left(\frac{x^{2}}{a^{2}}+\frac{y^{2}}{b^{2}}\right)}$

, where.

$z \quad$ autocorrelation value

$x$ horizontal point shift

$y$ vertical point shift

a horizontal point spread coefficient

$b$ vertical point spread coefficient

, with $z$ a dimensionless number between -1 and +1 , and grid points as the unit of measurement for $x, y, a$, and $b$ in Lagrangian coordinates.
Table 1 Calculation settings in LaVision DaVis

\begin{tabular}{ll}
\hline Subset size & $13,19,27,37$ pixels \\
Step size & 5 pixels \\
Correlation method & sum-of-differential (and relative-to-first) \\
Minimum number of valid pixels & $50 \%$ \\
Pyramid levels & 1 \\
Maximum iterations & 20 \\
Epsilon & 0.001 \\
Threshold for correlation value & 0.1 \\
Threshold for confidence margin & 0.1 pixel \\
Subset shape & round \\
High accuracy interpolation & on \\
Outlier and smoothing filters & off (except when otherwise stated) \\
Fit function & affine
\end{tabular}


Fig. 3 a) Filtered noise in a section of the strain map. b) The corresponding autocorrelation of the noise field with the colored surface showing the fitted function and the points indicating the autocorrelation data
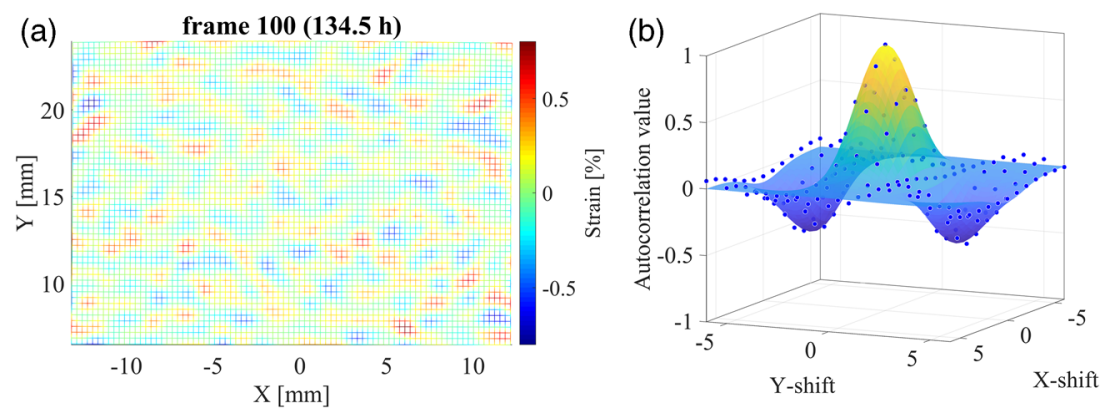

This theoretical function shape corresponds well with the experimental autocorrelation data calculated from the noise in the DIC strain measurement, as shown in Fig. 3b). Therefore, in addition to the standard deviation of the noise in the DIC strain measurement, the autocorrelation length was calculated from the noise component of the measured strain field. The two-parameter fit using equation (1) gives the least-squares fit coefficient $\boldsymbol{b}$ for vertical point spread. This value was then multiplied by the elongated grid spacing in pixels at that specific point in time to obtain a length characterizing the spatial resolution of the DIC measurement in pixels in the vertical direction.

\section{Comparison to Sample 14 of the DIC Challenge}

The DIC Challenge [18] provides test images for comparing the spatial resolution of different DIC codes. In particular, for Sample 14, sinusoidally varying "commanded" displacements with known constant amplitude and decreasing wavelength in horizontal direction (which results in increasing strain as the wavelength decreases) are imposed on the images. In the DIC Challenge paper, a relatively small attenuation of the measured strain variation amplitude is used as the threshold for considering the strain variations below the spatial resolution of the DIC measurement. Thus the DIC Challenge paper approached the concept of spatial resolution as answering the question "Does this DIC measurement accurately measure the strain amplitude, for strain variations at this wavelength?" This is not quite the same as answering the question "Are strain variations of this wavelength and amplitude detectable with this DIC measurement?" The combination of noise amplitude and autocorrelation length is directly suitable for answering the latter question, but not the first one.

However, for a rough comparison, the attenuation of the commanded displacement variations may be compared with the effect of filtering the commanded displacements by convolution with a Gaussian kernel of the appropriate width to result in the same autocorrelation length for originally uncorrelated noise. Thus, DIC was repeatedly performed on Sample 14 L5 of the DIC Challenge, with the same settings as for the experimental images, varying the subset size from 13 to 55 pixels. The larger the subset size and the smaller the wavelength of the imposed displacements, the more the measured displacements or strains will be attenuated from the imposed ones due to spatial filtering. For each of the DIC measurements with different subset sizes, the noise amplitude and autocorrelation length were extracted in horizontal direction from the horizontal component of the measured strain field, from the left side of Sample 14 L5 (x-position 50-550 pixels), which contains no or very small strains. The small strains were compensated by the local regression smoothing. The commanded displacements were then convolved with a Gaussian kernel given in equation (2), where the kernel width is defined by the autocorrelation length. The simultaneous attenuation of the commanded displacements by the DIC measurement and by convolution with the autocorrelation length were then plotted with the Matlab script of the DIC Challenge [21].

$G(x, w)=\frac{\sqrt{2}}{w \sqrt{\pi}} e^{-\frac{2 x^{2}}{w^{2}}}$

, where.

$x$ horizontal coordinate

$w$ kernel width defined by the autocorrelation length of the DIC measurement

For the case of convolving sinusoidally varying commanded displacements with a Gaussian kernel, when the sine wavelength $\lambda=2 \pi / k$ and amplitude $\alpha$ are constant:

$u(k, x)=\alpha \sin (k x)$

, the attenuation may be calculated analytically:

$\bar{u}(k, x, w)=\alpha e^{-\left(\frac{1}{8} k^{2} w^{2}\right)} \sin (k x)$

In the DIC Challenge paper, the spatial resolution is characterized by the wavelength of the sinusoidally varying displacement field for which the measured displacement variation amplitude or strain variation amplitude reaches an arbitrarily chosen attenuation threshold. The 
attenuation ratio $R$ for both displacements and strains equals to the exponential in equation (4):

$R(k, w)=e^{-\left(\frac{1}{8} k^{2} w^{2}\right)}=e^{-\left(\frac{\pi^{2} w^{2}}{2 \lambda^{2}}\right)}$

Thus, for this specific case there is an exact relationship between the metric of spatial resolution in the DIC Challenge paper and the autocorrelation length used in this paper. Although this relationship does not necessarily hold for DIC measurements in general, solving equation (5) for the wavelength results in a conversion factor that can be used to estimate the spatial resolution obtained using the procedure of the DIC Challenge from the autocorrelation length obtained using the procedure in this paper, or vice versa:

$\lambda_{\text {est }}(R, w)=\frac{\pi}{\sqrt{-2 \ln (R)}} w$

For the threshold of $R=0.95$ proposed in the DIC challenge paper for displacements, this results in $\lambda_{\text {est }}=9.809 w$, and for the value of $R=0.90$ used for strains in $\lambda_{\text {est }}=6.844 \mathrm{w}$.

\section{Results and Discussion}

\section{Experimental Results}

It is well established, that there is a trade-off between spatial resolution and precision, which governs and which is affected by the choice of subset size in relation to quality of the pattern and test conditions when analysing DIC measurements. Therefore, to assess the impact of methods to improve the spatial resolution or reduce the noise level in a DIC measurement, it is necessary to characterize that trade-off. Figure 4 shows the reduction in noise of a strain measurement when the test conditions are improved or the subset size is increased. In addition to the amplitude of the noise, the wavelength of the noise is affected by the measurement conditions and the subset size. In the absence of an imposed displacement field with variations in strain or displacement of well-defined length scale, the length scale of the noise in the measurement was determined, and used as a proxy for the spatial resolution of the measurement.

Figure 5 plots the extracted autocorrelation length versus the noise level for the three different specimens. The autocorrelation length correlates inversely with the noise level showing a logarithmic increase in noise when the subset size is reduced. The entire trade-off curves are shifted towards less noise by patterning and by diffuse lighting, enabling detection of smaller variations in strain. However, a simultaneous shift of the autocorrelation values to the right is observed. This is most likely explained by extraneous noise components in the worse measurements, with smaller wavelength than the autocorrelation length, which will shift the autocorrelation length towards smaller values. This shift is more visible with noisier data. The different length scale of the trade-off curve for specimen FSW-P-D, when compared to the EBW specimens, is most likely explained by the slightly different camera distance from the specimen.

A comparison of the sum-of-differential and relative-tofirst correlation methods is presented in Fig. 6 with data for frames 100, 200, and 300 for the specimen FSW-P-D. The respective points in time are $134.5 \mathrm{~h}, 270.3 \mathrm{~h}$, and $406.2 \mathrm{~h}$, and the mean strain over the gauge length is $6 \%, 15 \%$, and $26 \%$ without significant strain localization. Signal-to-noise ratios for the mean strain, when using sum-of-differential and subset size 27 , are $49.5,60.5$, and 61.5 , respectively. It is worth pointing out that if the signal of interest is the localization of deformation, with onset after some time and increasing amplitude, the signal to noise ratio improves even though the noise level increases. It is evident from Fig. 6 that relativeto-first accumulates less noise, but still the noise level increases similarly with added images in the series regardless of the correlation method. The deterioration of noise level is probably explained by increasing residual differences between deformed and initial images after the deformation is accounted for by the subset shape function, and how those affect the numerical minimisation of the residual by varying the deformation within the DIC algorithm. The deterioration of the spatial resolution as the experiment progresses is a result of elongation of the grid spacing, since the vertical point spread coefficient of the autocorrelation peak was multiplied by the elongated grid spacing.

It should be noted, that due to the non-linearity of the DIC calculation, its spatial filtering effect may be significantly different from the linear approximation of convolution with a point spread function. As a result, especially for unreasonably large subset sizes, the results of a DIC calculation may contain noise components with smaller wavelength than the autocorrelation length, and the spatial resolution may be smaller than expected from the subset size. For example, it can be seen in open implementations of DIC algorithms that under certain conditions with discontinuous displacement fields the cost function is bimodal, and the measured displacement discontinuously jumps when the smaller peak becomes the larger one. Likewise, the minimum in the cost function becomes less sharp when it is further away from zero - for example by not normalizing the image intensities - allowing spurious noisedominated terms in the correlation to contribute more. These noise components will shift the autocorrelation length towards smaller values, and it seems that the summation of small displacements amplifies this effect, since the autocorrelation lengths obtained for sum-of-differential measurements, when compared to relative-to-first measurements, are smaller when using the same subset size (Fig. 6). To investigate this in detail is beyond the scope of this paper and it would require low- 
Subset size 19

Subset size 27

Subset size 37
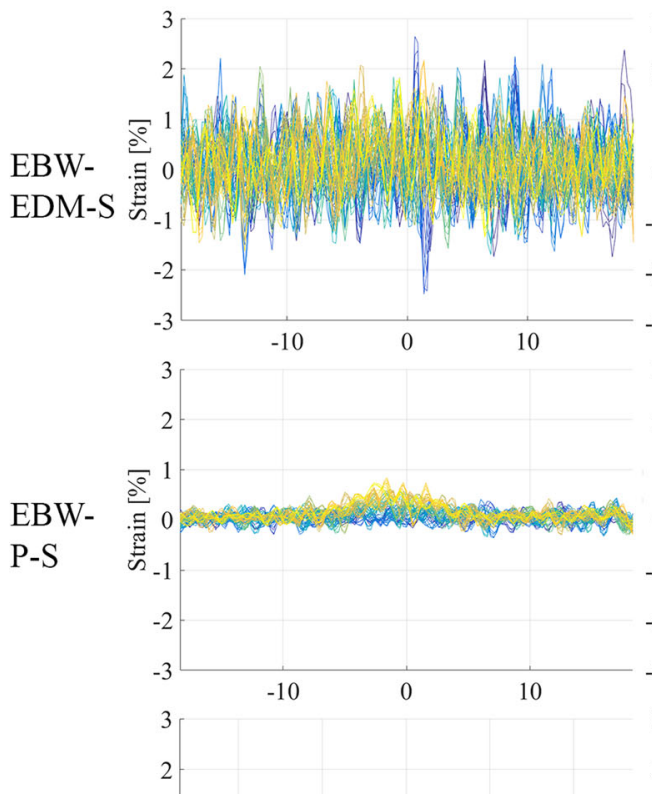

FSW-
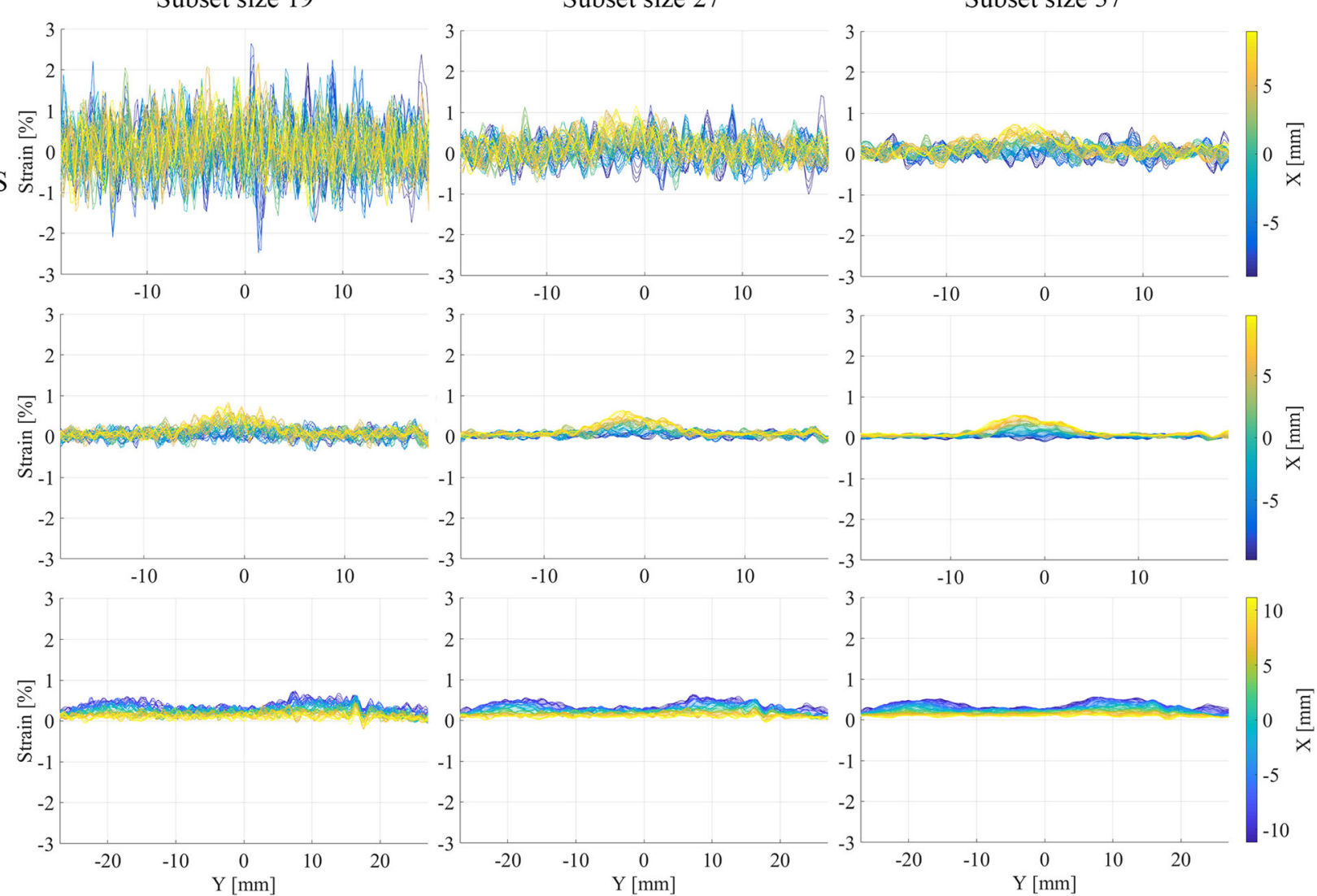

Fig. 4 Small variations in strain in comparison to the noise level of the measurement for the three different specimens. The subset size increases from left to right showing the reduction in noise and increase in wavelength of the noise. $\mathrm{X}$ and $\mathrm{Y}$ indicate positions across and along the gauge length of the tensile specimen, respectively

level access to the DIC algorithm and intermediate results. However, if such noise deteriorates the data, the measurement can trivially be improved by smoothing the displacement field with filters smaller than the spatial resolution of the measurement. Thus, the autocorrelation length is an approximate measure of all of the noise components, including any extraneous

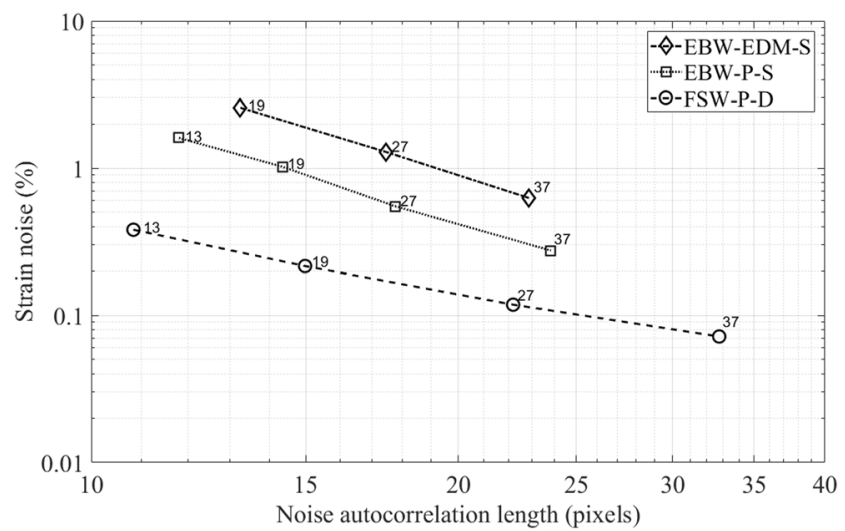

Fig. 5 Trade-off curves of strain noise and noise autocorrelation length for frame 100. Patterning (EBW-P-S, FSW-P-D) and diffuse lighting (FSW-P-D) shift the entire trade-off curves towards less noise. The subset size varies from 13 to 37 pixels, except for EBW-EDM-S, with which DIC failed to correlate with subset size 13 noise which might not be dependent on the spatial resolution of the measurement. Therefore, the autocorrelation length should be taken as an upper bound of the spatial resolution, with the given test conditions and computational factors.

It is a choice of the experimentalist to aim for better noise level or better spatial resolution of a DIC measurement. The DIC software often provides filters for the improvement of the

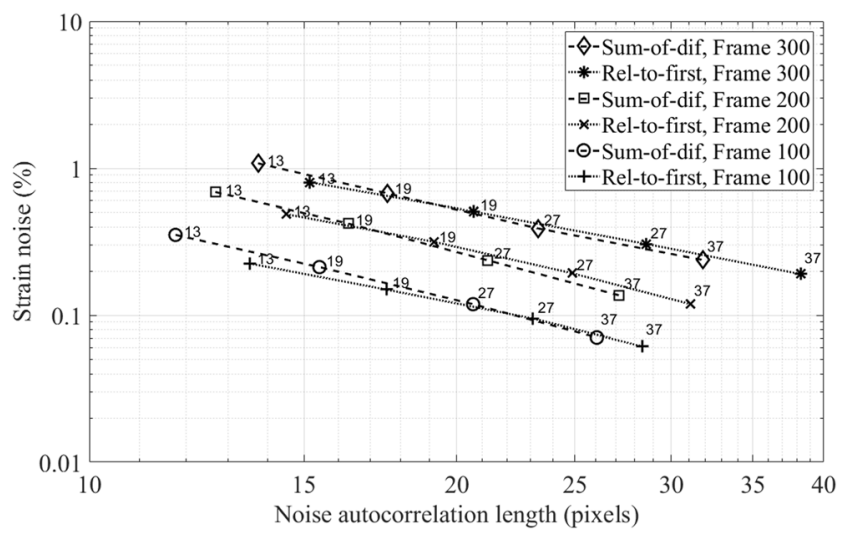

Fig. 6 Comparison of relative-to-first and sum-of-differential correlation methods with data for frames 100, 200, and 300 for the specimen FSW-PD. The strain noise and autocorrelation length deteriorate with added images in the series. The subset size varies from 13 to 37 pixels 
noise level, but this happens at the expense of the spatial resolution. The effect of the filters on the autocorrelation length is shown in Fig. 7. The filters clearly compress the trade-off curve towards larger autocorrelation lengths while the noise is reduced. This is especially evident with small subsets, which suffer from high level of noise initially. Larger subsets, on the other hand, do not benefit from the filters, since they already perform as initial smoothing filters in the DIC calculation process.

The best data quality was achieved by using the optimized DIC pattern and indirect diffuse lighting, which minimized specular reflections from the specimen surface. Alternatively, a white pattern background would have eliminated point-like specular reflections from facets on the grains of copper or the EDM-roughened surface, but possibly not a glare from the spotlight. Thus, using the diffuse lighting or a white coating would nearly eliminate contrast from the specimen with the as-cut EDM surface. However, copper oxide was used for patterning because it adheres to the copper surface better than a paint or foil would have, and this process is incompatible with applying a white background.

\section{Sample 14 of the DIC Challenge}

Since the metric of spatial resolution in the DIC Challenge paper [18] is different than the metric proposed in this paper, a direct comparison of the obtained values is not generally valid. In addition, the images provided in the DIC Challenge did not include strain variations with sufficiently short wavelength to really challenge the DIC codes with respect to the detectability of the commanded strain variations. However, for the special case of spatial filtering by convolution with a Gaussian function, the relationship between the two metrics is well-defined by the shape of the Gaussian function, and each of the methodologies can be compared with that common reference. Therefore, we compared the measured strains with

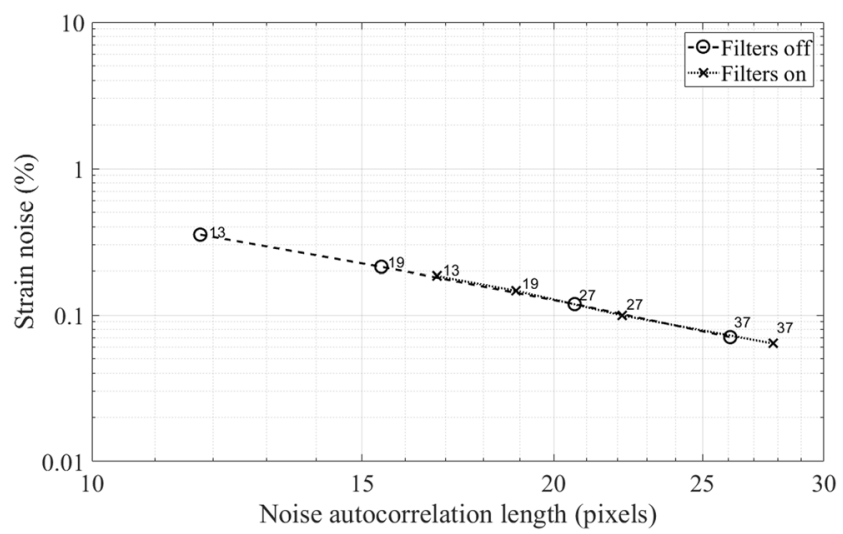

Fig. 7 Filters in the DIC software reduce the strain noise while worsening the autocorrelation length. The data is for the patterned FSW-P-D specimen with indirect diffuse lighting. The subset size varies from 13 to 37 pixels both the commanded strains and the commanded strains smoothed by a convolution that for random noise results in the same autocorrelation length as the noise autocorrelation length of the measured strains. The obtained curves are shown in Fig. 8. DIC measurement with increasing subset size results in increasing autocorrelation length of the measured strains, as shown in Fig. 8a), as well as increasing attenuation of the measurement from the commanded strains at shorter wavelengths (Fig. 8b-f). Convolution of the commanded strains in Fig. 8b-f), with increasingly broad point spread functions, corresponding to the same strain noise autocorrelation lengths from Fig. 8a), results in good agreement for the attenuation of the strain variations. Thus it seems that for this DIC code with these patterns and these parameters, the relationship that holds between these two metrics of spatial resolution that holds for convolution with a Gaussian point spread function, approximately holds for the spatial filtering that happens in the DIC measurement, as well. Table 2 compares these two metrics, with numerical values for the autocorrelation length $w$, the corresponding attenuation threshold wavelength $\lambda_{\text {est }}=$ $6.844 w$ from equation (6), and the spatial resolution that the Matlab script of the DIC Challenge returns for the strains measured in this paper.

\section{Summary and Conclusions}

This paper consists of three main parts: 1) Improvement of DIC test conditions by optimized patterning of copper tensile specimens and diffuse lighting, 2) quantifying the improvements by extraction of noise level and autocorrelation length of the noise, and 3) situating the strain noise autocorrelation length as a proxy for the spatial resolution of the measurement, by analysis of images with known imposed displacements [18].

The copper tensile specimens were patterned with the aid of a photolithographic method by using black copper oxide as the patterning medium. The advantage of copper oxide is that it adheres to the surface better than a paint or foil would have. This enables full-field measurements of large deformations in copper tensile specimens, as well as detection of smaller variations in strain due to improved precision of the measurement.

The noise level of the measurements was calculated from a section of the strain map by subtracting a fitted local average strain, and by taking the standard deviation of the noise extracted this way. There is an intrinsic length scale of the noise. This length scale was extracted by autocorrelation of the noise field, and by determining the spread of the autocorrelation peak. The value was then multiplied by the elongated grid spacing and used as a proxy for the spatial resolution of the measurement. The method requires a region with fairly smooth strains for the extraction of the noise since the local 
(a)

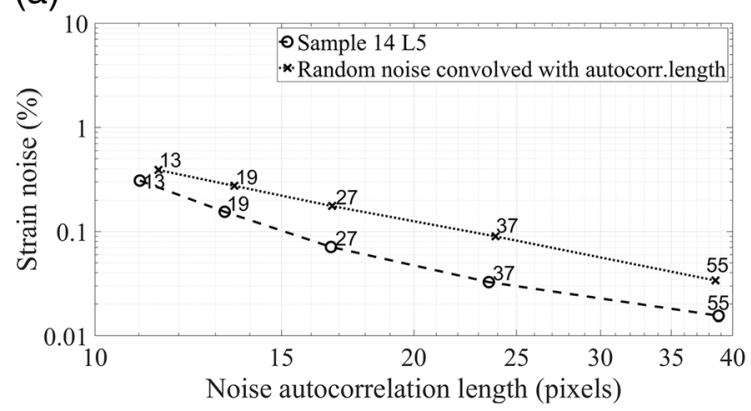

(c)

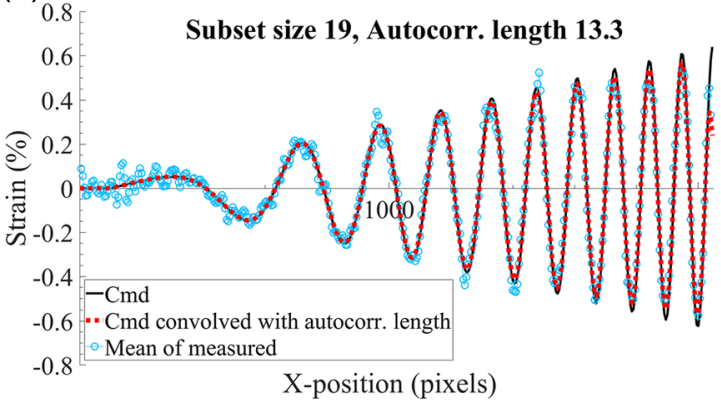

(e)

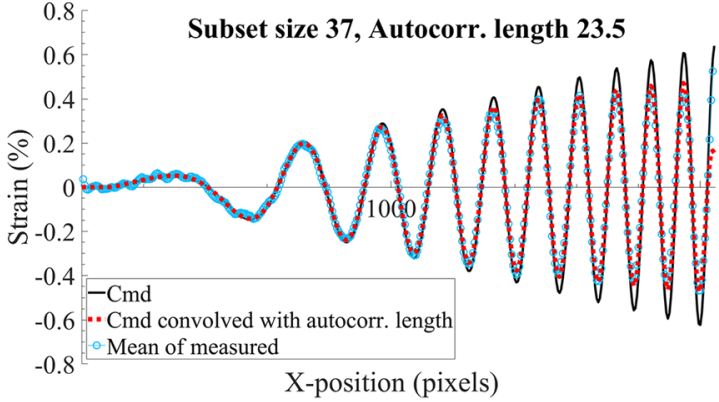

(b)

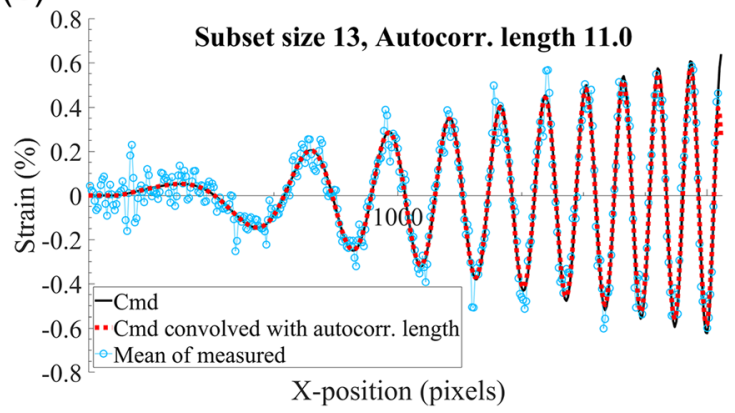

(d)

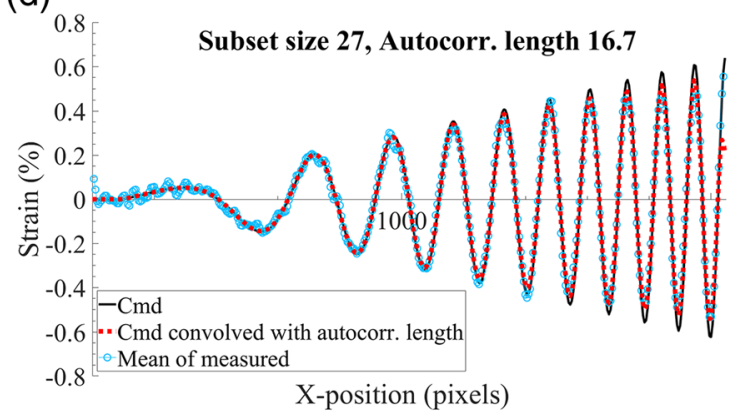

(f)

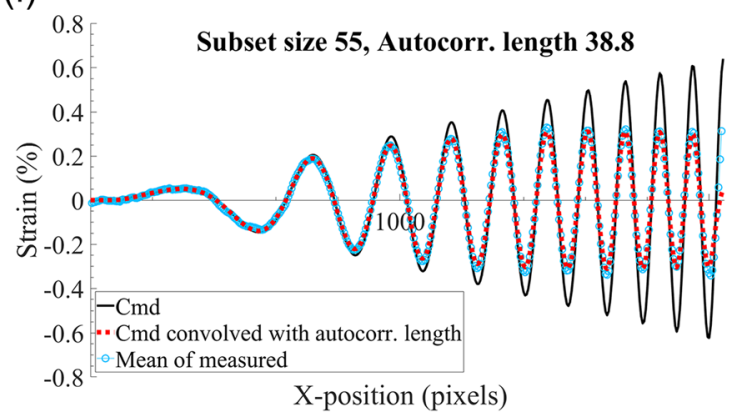

Fig. 8 a) Measured strain noise and noise autocorrelation length of Sample 14 L5 [18], as well as, random noise convolved in two dimensions with increasingly broad point spread functions corresponding to the noise autocorrelation lengths. b-f) Attenuation of strains in Sample 14 L5 due to the DIC measurement and due to convolution of the commanded strains with kernel widths corresponding to the autocorrelation lengths for the different subset sizes

regression smoothing can only compensate for a limited amount of strain localization. In addition, the size of the extracted noise field should be significantly larger (>10x) than the subset size for reliable extraction of the noise wavelength

Table 2 Autocorrelation lengths (w) of the DIC measurement as a function of the subset size, converted to attenuation threshold wavelength $\left(\lambda_{\text {est }}\right)$ for comparison with the metric of spatial resolution of the DIC Challenge paper $\left(\lambda_{\text {thr }}\right)$

\begin{tabular}{cllc}
\hline Subset size & $w$ & $\lambda_{\text {est }}($ at $R=0.90)$ & $\lambda_{\text {thr }}($ at $R=0.90)$ \\
\hline 13 & 11.0 & 76 & $\mathrm{n} / \mathrm{a}$ \\
19 & 13.3 & 91 & 105 \\
27 & 16.7 & 114 & 126 \\
37 & 23.5 & 161 & 166 \\
55 & 38.8 & 265 & 252 \\
\hline
\end{tabular}

by autocorrelation. Comparing the spatial filtering, that happens in the DIC measurements discussed here, to convolution with a Gaussian point spread function, the Gaussian width that results in the same strain noise autocorrelation length also gives comparable attenuation of known imposed strains.

The results obtained by the above method quantify the trade-off between the minimum magnitude of localized strain (noise) and minimum extent of localized deformation (spatial resolution) that can be detected when varying the calculation parameters and test conditions. The method may be used to estimate the spatial resolution of a displacement or strain field, based only on the displacement data-set itself, without any knowledge at all about how it was measured. This spatial resolution value includes the combined effects of the pattern, tests conditions, and computational factors. Thus the method may be used for assessment of improvements in a DIC measurement when looking for optimum test conditions and 
parameters, or for comparing DIC measurements with other full-field measurement methods.

Acknowledgements Open access funding provided by Aalto University. The authors would like to thank the Finnish national nuclear safety program (KYT2018) for funding the research, Posiva Oy (Finland) for the EBW welds, and Swedish Nuclear Fuel and Waste Management Co. (SKB) for the FSW welds. The contribution of Kim Widell in helping with tensile testing, and Mykola Ivanchenko and Lauri Luumi for preliminary work done in the project is also highly appreciated.

Open Access This article is distributed under the terms of the Creative Commons Attribution 4.0 International License (http:// creativecommons.org/licenses/by/4.0/), which permits unrestricted use, distribution, and reproduction in any medium, provided you give appropriate credit to the original author(s) and the source, provide a link to the Creative Commons license, and indicate if changes were made.

\section{References}

1. Sutton M, Orteu J, Schreier H (2009) Image correlation for shape, motion and deformation measurements. Springer Science, New York

2. Wang Y, Sutton M, Bruck H, Schreier H (2009) Quantitative error assessment in pattern matching: effects of intensity pattern noise, interpolation, strain and image contrast on motion measurements. Strain 45:160-178

3. Bornert M, Brémand F, Doumalin P, Dupré J, Fazzini M, Grédiac M, Hild F, Mistou S, Molimard J, Orteu J, Robert L, Surrel Y, Vacher P, Wattrisse B (2009) Assessment of digital image correlation measurement errors: methodology and results. Exp Mech 49: 53-370

4. Dupré J, Bornert M, Robert L, Wattrisse B (2010) Digital image correlation: displacement accuracy estimation. EPJ Web Conf 6: 31006

5. Amiot F, Bornert M, Doumalin P, Dupré J, Fazzini M, Orteu J, Poiâne C, Robert L, Rotinat R, Toussaint E, Wattrisse B, Wienin J (2013) Assessment of digital image correlation measurement accuracy in the ultimate error regime. Strain 49:483-496

6. Pan B, Qian K, Xie H, Asundi A (2008) On errors of digital image correlation due to speckle patterns. In He X, Xie H, Kang Y, (eds.) ICEM 2008: International Conference on Experimental Mechanics 7375:73754Z
7. Collette A, Sutton M, Miney P, Reynolds A, Xiaodong L, Colavita P, Scrivens W, Luo Y, Sudarshan T, Muzykov P, Myrick M (2004) Development of patterns for nanoscale strain measurements. Nanotechnology 15:1812-1817

8. Scrivens W, Luo Y, Sutton M, Collette S, Myrick M, Miney P, Colavita P, Reynolds A, Li X (2007) Development of patterns for digital image correlation measurements at reduced length scales. Exp Mech 47:63-77

9. Kammers A, Daly S (2011) Small-scale patterning methods for digital image correlation under scanning electron microscopy. Meas Sci Technol 22:125501

10. Raiko H (2012) Canister design 2012. Posiva report 2012-13

11. Bossuyt S (2013) Optimized patterns for digital image correlation. Proceedings of the 2012 Annual Conference on Experimental and Applied Mechanics 3

12. Lecompte D, Smits A, Bossuyt S, Sol H, Vantomme J, Van Hemelrijck D, Habraken A (2006) Quality assessment of speckle patterns for digital image correlation. Opt Lasers Eng 44:11321145

13. Fazzini M, Mistou S, Dalverny O, Robert L (2010) Study of image characteristics on digital image correlation error assessment. Opt Lasers Eng 48:335-339

14. Ning J, Braxton V, Wang Y, Sutton M, Wang Y, Lessner S (2011) Speckle patterning of soft tissues for strain field measurement using digital image correlation: preliminary quality assessment of patterns. Microsc Microanal 17:81-90

15. Triconnet K, Derrien K, Hild F, Baptiste D (2009) Parameter choice for optimized digital image correlation. Opt Lasers Eng 47:728-737

16. Cofaru C, Philips W, Paepegem W (2010) Improved NewtonRaphson digital image correlation method for full-field displacement and strain calculation. Appl Opt 49:6472-6484

17. Schreier H, Sutton M (2002) Systematic errors in digital image correlation due to undermatched subset shape functions. Exp Mech 42:303-310

18. Reu PL, Toussaint E, Jones E, Bruck HA, Iadicola M, Balcaen R, Turner DZ, Siebert T, Lava P, Simonsen M (2018) DIC challenge: developing images and guidelines for evaluating accuracy and resolution of 2D analyses. Exp Mech 58:1067-1099. https://doi.org/ 10.1007/s11340-017-0349-0

19. Jones E, Iadicola M (eds) (2018) A good practices guide for digital image correlation. Int Digit Image Correlation Soc. https://doi.org/ 10.32720/idics/gpg.ed1

20. Pihlajamäki T (2017) Characterization of strain aging with full-field strain measurements, MSc. Thesis. Aalto University, Finland

21. Reu PL private communication, January 2019

Publisher's Note Springer Nature remains neutral with regard to jurisdictional claims in published maps and institutional affiliations. 